\title{
A study on cholestasis in infants less than six months of age presenting to Lady Ridgeway Hospital for Children, Colombo
}

\author{
*Mohamed Infaq Mohamed Luthufdeen ${ }^{1}$, Samantha Waidyanatha ${ }^{2}$ \\ Sri Lanka Journal of Child Health, 2016; 45(1): 34-37
}

\begin{abstract}
Objectives: To determine the common clinical presentations, common aetiological factors, average age at presentation and possible means of early detection of cholestasis by primary health care workers.
\end{abstract}

Method: This prospective, descriptive study analyzed 60 infants less than six months old who presented to Lady Ridgeway Hospital from October 2013 to January 2015 with evidence of cholestasis.

Results: The common clinical features were jaundice (100\%), hepatomegaly $(83.3 \%)$, pale stools $(76.6 \%)$, dark urine $(61.6 \%)$ and splenomegaly $(56.6 \%)$. The common causes were idiopathic neonatal hepatitis (INHS) (26.6\%) and biliary atresia (26.6\%). Nine cases of INHS were transient. Sepsis (15\%), multifactorial $(13.3 \%)$ and cytomegalovirus infection $(6.6 \%)$ were the other frequent causes. The less frequent causes were Alagille syndrome (3.2\%), birth asphyxia (1.6\%), progressive familial intrahepatic cholestasis (PFIC) (1.6\%), biliary sludge due to haemolysis $(1.6 \%)$, intestinal obstruction $(1.6 \%)$ and multiple small gall bladder calculi (1.6\%). The average age at presentation of infants with cholestasis was 64.2 days and the mean age at presentation of infants with cholestasis who were eventually diagnosed as biliary atresia was 81.8 days.

${ }^{1}$ Specialty Doctor in Paediatrics, The Queen Elizabeth Queen Mother Hospital, Margate, Kent, United Kingdom, ${ }^{2}$ Consultant Paediatrician, Lady Ridgeway Hospital for Children, Colombo, Sri Lanka

*Correspondence: Infaaq555@yahoo.com

(Received on 13 April 2015: Accepted after revision on 22 May 2015)

The authors declare that there are no conflicts of interest

Personal funding was used for this project.

Open Access Article published under the Creative Commons Attribution CC-BY (cC) (P)
Conclusions: Jaundice, hepatomegaly and pale stools were the common clinical features on presentation. INHS and biliary atresia were the common causes of cholestasis. There was a significant delay in the presentation of cases of cholestasis due to primary health care failure.

DOI: http://dx.doi.org/10.4038/sljch.v45i1.8083

(Key words: Neonatal cholestasis, biliary atresia, idiopathic neonatal hepatitis syndrome, primary healthcare workers, stool colour code)

\section{Introduction}

As extrahepatic biliary obstruction and neonatal hepatitis syndrome carry high mortality and morbidity rates if not treated early in life, it is important to diagnose these conditions early in order to have a good outcome ${ }^{1}$. Idiopathic neonatal hepatitis (INHS) and biliary atresia are the most frequent causes of cholestatic jaundice in the first months of life $\mathrm{e}^{2,3,4}$. Syndrome of neonatal hepatitis has diverse causes. Idiopathic form, sepsis/urinary tract infection, genetic diseases of metabolism and congenital infections are relatively common causes compared to toxic causes, post-haemolytic states, neonatal acute hepatic necrosis, parenteral nutrition, chromosomal anomalies, familial syndromes etc ${ }^{4}$. Measurement of serum conjugated bilirubin and observation of stool colour are important screening strategies to detect cholestasis early by primary care providers $^{5}$. Diagnosis of extra hepatic biliary atresia as early as possible (younger than 60 days of age) is the key determinant to successful hepatic portoenterostomy ${ }^{6}$.

\section{Objectives}

To determine common clinical presentations, common aetiological factors, the average age at presentation of infants with cholestasis, less than six months old who presented to Lady Ridgeway Hospital and to address the possible means of early detection of cholestasis by primary health care workers. 


\section{Method}

This prospective, descriptive study analyzed 60 infants, less than six months old, with clinical or biochemical evidence of cholestasis, who presented to Lady Ridgeway Hospital. The study commenced in October 2013 and continued until the sample size of 60 was achieved in January 2015. After taking informed written consent from a parent or a guardian, data were extracted from the bed head tickets as well as by direct questioning using a questionnaire.

\section{Results}

The frequencies of common clinical features for cholestasis in study population are shown in table 1 .

Table 1

Frequencies of common clinical features for cholestasis in study population $(n=60)$

\begin{tabular}{|l|c|}
\hline \multicolumn{1}{|c|}{ Clinical feature } & Number (\%) \\
\hline Jaundice & $60(100)$ \\
Hepatomegaly & $50(83.3)$ \\
Pale stool & $46(76.6)$ \\
Dark urine & $37(61.6)$ \\
Splenomegaly & $34(56.6)$ \\
\hline
\end{tabular}

The stools of all infants who were diagnosed as biliary atresia were pale since early neonatal period. Among 46 cases of cholestasis with pale stools, 35 (76\%) cases presented late due to primary health care failure and the remaining $11(24 \%)$ were identified early because they were hospitalized during the neonatal period due to other problems prior to the development of overt cholestasis.

The frequencies of different causes for cholestasis in the study population are shown in table 2 .

Table 2

Frequencies of different causes for cholestasis in study population

\begin{tabular}{|l|c|}
\hline Causes & No. (\%) \\
\hline Idiopathic neonatal hepatitis (INHS) & $16(26.6)$ \\
Biliary atresia & $16(26.6)$ \\
Hepatitis purely related to sepsis & $09(15.0)$ \\
Multifactorial & $08(13.3)$ \\
CMV infection & $04(06.6)$ \\
Alagille syndrome & $02(03.3)$ \\
Birth asphyxia & $01(01.6)$ \\
Progressive familial intrahepatic & \\
cholestasis & $01(01.6)$ \\
Biliary sludge due to hemolysis & $01(01.6)$ \\
Intestinal obstruction & $01(01.6)$ \\
Multiple small gall bladder calculi & $01(01.6)$ \\
\hline
\end{tabular}

Nine cases of INHS were identified as transient idiopathic neonatal hepatitis syndrome. One of the cases with sepsis also had multiple small gall bladder calculi and a patient with biliary atresia co-existed with cytomegalovirus (CMV) infection. Out of the eight infants with multiple reasons for cholestasis, sepsis was present in all 8, birth asphyxia in 3, dysmorphism in 3 , intestinal obstruction in 2, prematurity and intrauterine growth restriction in 1, multiple small gall bladder calculi in 1 and possible metabolic disorder in 1 . Twelve of the 16 cases of idiopathic neonatal hepatitis syndrome, had TORCH screening. Other four infants had not undergone any testing for CMV infection, either serological or PCR testing.The liver biopsies of two cases were suggestive of progressive familial intrahepatic cholestasis ( PFIC). However, the diagnosis of one of these later turned out to be CMV hepatitis.

The study showed that there was a significant delay in the presentation of cases of cholestasis to Lady Ridgeway Hospital, the average age of presentation being 64.2 days. Infants who were diagnosed as biliary atresia presented at the mean age of 81.8 days, and the majority of these cases $(68.8 \%)$ presented after 60 days. Twelve (75\%) of infants with biliary atresia underwent the Kasai procedure at the mean age of 83.3 days. The other $25 \%$ were very late presenters and, therefore, not suitable for the surgery on presentation. Furthermore, the study revealed, six out of $12(50 \%)$ cases of biliary atresia who underwent Kasai surgery had an under-one year mortality rate of $50 \%$ (surgery was performed after 60 days of age on all of them). While two cases of biliary atresia after the Kasai surgery showed progressive liver disease (surgeries done on day 83 and 57), one showed no progression and the other showed some improvement after the surgery (surgeries done on day 90 and day 66 respectively). The remaining two cases underwent surgery recently and, therefore, it is too early to comment.

Three cases of idiopathic neonatal hepatitis syndrome died of progressive liver failure. While two showed progression of liver disease one showed no progression and one case was lost to follow up. Among the nine septic infants all survived except one. Out of four CMV hepatitis cases, one succumbed and one showed progression of liver disease. However, two cases of CMV hepatitis improved spontaneously.

Retrospective review of ultrasonography for diagnosis of biliary atresia based on the appearance of the gall bladder revealed a false negative result of $14.3 \%$ (2 in 14) and false positive result of $39.1 \%$ (9 
in 23). The triangular cord sign was positive only in one case of biliary atresia. Liver biopsies did not show the added advantage of the decisive information as only 1 out of $15(6 \%)$ liver biopsies was conclusive $(6 \%)$ for a particular diagnosis while the HIDA scan was somewhat better with the success rate of $71.5 \%$ in diagnosing and excluding biliary atresia. Intra operative cholangiogram (IOC) was the best among the diagnostic tools with the accuracy of $100 \%$ in diagnosing and excluding biliary atresia according to the tests performed on seven infants of the study population.

\section{Discussion}

This study showed that the stools of all infants who were eventually diagnosed as biliary atresia were pale since the early neonatal period. The clinical feature noted to be sensitive for extra hepatic biliary atresia was the presence of acholic or varying acholic stools on admission. There was no single clinical feature with sufficient sensitivity and specificity to differentiate extra hepatic biliary atresia from other causes of neonatal cholestasis. In a study done in Malaysia in 146 infants with cholestasis, the three most common causes were INHS $(n=63,43 \%)$, extra hepatic biliary atresia $(n=35,24 \%)$ and congenital cytomegalovirus hepatitis $(\mathrm{n}=13,9 \%)^{7}$. Our study also showed the same pattern, but the cases of idiopathic neonatal hepatitis syndrome were not fully evaluated with complete TORCH screening, maybe due to the difficulties and the cost involved with the tests. In only eight cases of neonatal hepatitis syndrome was a complete workout for CMV infection done and five of them became positive for CMV infection. One of these infants who were positive for CMV infection coexisted with biliary atresia. It is important to have a well-organized and structured approach to detect TORCH related cholestasis, in particular CMV hepatitis because according to this study, the cases of CMV related cholestasis were underdiagnosed. Moreover, investigating and diagnosing of metabolic disorders like PFIC is also a difficult task because of financial constraint and non-availability of facilities.

Most patients in whom no aetiology was found were considered to have transient neonatal cholestasis by some authors. Transient neonatal cholestasis was characterized by: early-onset cholestasis, absence of a known cause of neonatal cholestasis, normalization of clinical and biochemical parameters during followup, and no history of some neonatal injurious events such as asphyxia, sepsis, total parenteral nutrition ${ }^{8}$. As this study also had shown transient neonatal cholestasis as one of the major reasons for cholestasis in infants, it is important to consider this as a possible reason in cases, when there is clinical improvement and no obvious cause is found for the cholestasis. This would avoid doing unnecessary major investigations.

Delay in referral of cases of neonatal hepatitis syndrome to appropriate centres is still a major problem in many parts of the world ${ }^{9,10}$. It was shown in a study done in India that there was a considerable delay in the referral of patients to appropriate centres for management. A delay of $120.8+/-60.5$ days in extra hepatic biliary atresia and $65.9+/-39.2$ days in neonatal hepatitis syndrome were documented ${ }^{9}$. In our study there was a significant delay in the presentation of cases of cholestasis to Lady Ridgeway Hospital with the average age being 64.21 days. Infants, who were eventually diagnosed as biliary atresia presented at the mean age of 81.75 days and the mean age of performing the Kasai procedure was 83.25 days. Moreover, this study has shown that a large number of the infants presented late despite having pale stools from the early neonatal period. The majority of these infants were evaluated by the primary health care workers. Therefore, it is presumed that infants with cholestasis presenting late, could have been minimized if a screening test like stool colour code system was available to parents and/or primary health care workers. Furthermore, the primary health care workers must be alerted when they deal with cases of infants with persistent jaundice for more than two weeks and/or with pale stool at any stage to refer them early to an appropriate centre. However, persistently clay coloured stools had a modest accuracy to differentiate extra hepatic biliary atresia from the neonatal hepatitis syndrome ${ }^{11}$. Studies have shown that introducing stool colour card screening system improved the outcome of extra hepatic biliary atresia by early identification and early institution of specific management ${ }^{12,13}$. This screening system might be especially effective in areas with a high proportion of late referrals ${ }^{12}$.

The most common presentations of cholestasis were jaundice, hepatomegaly, pale stool, dark urine and splenomegaly. The more frequent aetiological factors for cholestasis were idiopathic neonatal hepatitis syndrome and biliary atresia. Other frequent causes were sepsis related hepatitis and cholestasis with multiple problems where single aetiology could not be identified as to the cause of cholestasis. Furthermore, the study had shown a significant delay in the presentation of cases of cholestasis to Lady Ridgeway Hospital due to primary health care failure. It is, therefore, highly recommended to find an 
effective way to identify cholestasis early by parents and by primary health care workers. In this regard, a system like stool colour code on child health developmental record will be greatly beneficial. In addition, more facilities should be made available to the health care workers, particularly, at a tertiary care level to investigate these infants with cholestasis with complete TORCH screening and the metabolic investigations.

\section{References}

1. Keffler, Sue Kelly, Deirdre A, Powell, Judith, Green, Anne. Population screening for neonatal liver disease. A feasibility study. Journal of Pediatric Gastroenterology and Nutrition 1998; 27(3): 306-11. http://dx.doi.org/10.1097/000051761998090 00-00007

PMid: 9740202

2. Lippincott Williams \& Wilkins, Philadelphia. Guideline for the Evaluation of Cholestatic Jaundice in Infants: Recommendations of the North American Society for Paediatric Gastroenterology, Hepatology and Nutrition. Journal of Pediatric Gastroenterology and Nutrition 2004; 39:115-28.

http://dx.doi.org/10.1097/000051762004080 00-00001

3. M Najafi, AKhodadad, GhR Khatami, F Farahmand, GhHFalahi, F Motamed. Prevalence of different aetiology in neonatal cholestasis. Iranian Journal of Pediatrics 2006; 16(3):289-94.

4. Dragomir D, Popescu V. The neonatal hepatitis syndrome. Rev Pediatr Obstet Ginecol Pediatr 1990; 39(1):1-41.

PMid:2128714

5. Sokol RJ, Shepherd RW, Superina R, Bezerra JA, Robuck P, Hoofnagle JH. Screening and outcomes in biliary atresia:summary of a National Institutes of Health workshop. Hepatology 2007;46 (2):566-81. http://dx.doi.org/10.1002/hep.21790

PMid:17661405 PMCid:PMC3888317
6. Sangkhathat $\mathrm{S}, \quad$ Patrapinyokul S, Tadtayathikom K, Osatakul S. Perioperative factors predicting the outcome of hepatic porto-enterostomy in infants with biliary atresia. Journal of the Medical Association of Thailand 2003; 86(3):224-31. PMid:12757061

7. Lee WS, Chai PF. Clinical features differentiating biliary atresia from other causes of neonatal cholestasis. Annals Academy of Medicine Singapore 2010; 39 (8):648-54. PMid:20838708

8. Ciocca M, Alvarez F. Transient neonatal cholestasis. Arch Argent Pediatr 2011; 109(2):163-6.

PMid:21465076

9. Yachha SK, Mohindra S. Neonatal cholestasis syndrome: Indian scene. Indian Journal of Pediatrics 1999; 66(1Suppl): 946.

10. De Bruyne R, Van Biervliet S, VandeVelde $\mathrm{S}$, Van Winckel M. Clinical practice: neonatal cholestasis. European Journal of Pediatrics 2011; 170(3):279-84. http://dx.doi.org/10.1007/s00431-010-13638

PMid:21249394

11. Poddar U, Thapa BR, Das A, Bhattacharya A, Rao KL, Singh K. Neonatal cholestasis: differentiation of biliary atresia from neonatal hepatitis in a developing country. Acta Paediatr. 2009 Aug; 98(8):260- 4. http://dx.doi.org/10.1111/j.16512227.2009.0 1338.x

PMid:19469771

12. Tseng JJ, Lai MS, Lin MC, Fu YC. Stool color card screening for biliary atresia. Pediatrics 2011; 128(5):1209-15. http://dx.doi.org/10.1542/peds.2010-3495 PMid:22025588

13. Chang $\mathrm{MH}$. Screening for biliary atresia. Chang Gung Medical Journal 2006; 29(3): 231-3. PMid:16924883 